\title{
TISSUE STIFFNESS FROM TACTILE IMAGING
}

\author{
A.M. Galea, R.D. Howe
}

Division of Engineering and Applied Sciences, Harvard University, MA, USA

\begin{abstract}
Tactile imaging uses a passive pressure sensitive sensor to record the reaction pressure distribution on its surface. When pressed into and scanned over tissue of interest, it generates an image that is dependent on the mechanical properties and geometric distribution of the structures within the tissue. Since pathology is related to tissue stiffness, stiffness measurement would be of great use to provide insight into disease processes and an aid to diagnosis. We have developed an algorithm that inverts the tactile image and determines the salient features of the underlying tissue. The features of interest are the stiffness and depth of the background tissue and the stiffness and size of a round inclusion. A finite element model was constructed in order to simulate the tactile imaging process. The analysis was performed on models spanning an experimentally determined range of material properties. By analyzing each pressure frame and performing a least-squares fit between the pressure frames and the model features we were able to determine an inverse relationship to extract tissue stiffness from tactile frames.
\end{abstract}

Keywords - Palpation, imaging, tissue stiffness, inclusion.

\section{INTRODUCTION}

Tactile imaging systems can map the palpable extent of stiff lesions in soft tissue [1,2]. A two-dimensional array of pressure sensors are mounted on a rounded head that is pressed by hand into a tissue and scanned across the surface. Tactile imaging can be applied to breast cancer screening, to augment manual palpation. It is also potentially useful in surgery for locating lumps in the liver and lung, which commonly exhibit lesions as a discrete area of increased stiffness.

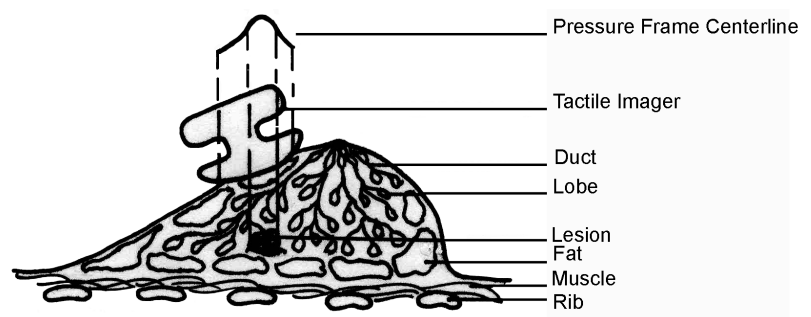

Figure 1. Obtaining tactile images on a breast. The image features are dependent on the stiffness and geometry of the underlying tissue.

Tactile image information is valuable in visualizing the characteristics of the underlying tissue, however the pressure patterns are not directly related to the absolute stiffness of the tissue imaged. Like the information gathered in manual palpation, the tactile information is a combination of tissue stiffness and the geometry in which it is distributed [3,4,5]. Lesion stiffness is correlated to pathology, so knowing the absolute stiffness of the underlying tissue would provide insight into disease processes as well as a better understanding of the properties of healthy tissue [6].

\section{FORMULATION}

A simple model of a round inclusion in homogenous tissue represents a broad spectrum of palpation conditions. For such a model, the features of interest are the inclusion size and stiffness, and the tissue depth and stiffness. We seek the inverse relation between the surface pressure information available to tactile imaging and the underlying tissue features.

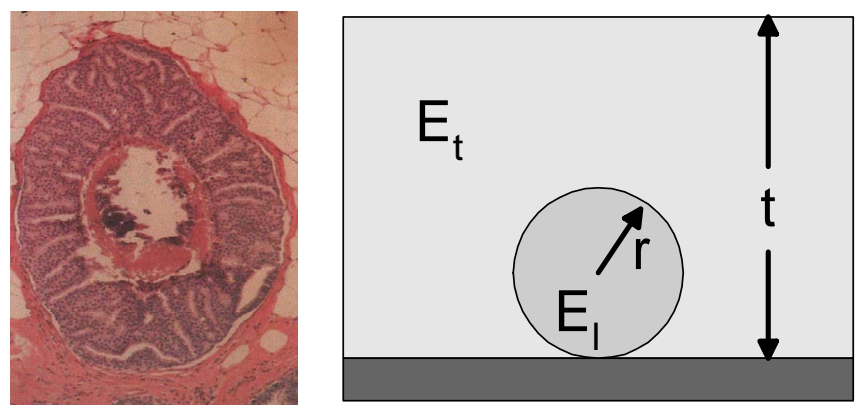

Figure 2. Left. Typical picture of breast cancer: ductal carcinoma in situ. The cancer $(<5 \mathrm{~mm}$ diameter in this case) is growing out of the underlying stiff glandular tissue into the surrounding soft fat [Photo courtesy of Dr. Susan Lester]. Right. Simple model of hard inclusion in soft tissue for our analysis. The features of interest are the lesion size $r$ and stiffness $E_{l}$, and the tissue depth $t$ and stiffness $E_{t}$.

\section{METHODOLOGY}

Performing plain strain analysis along a plane of symmetry of this model yields results applicable to a round inclusion such as a cyst or a discrete malignancy as well as to a cylindrical inclusion such as a vessel. Nonlinear finite element analysis was conducted in order to simulate tactile imaging and obtain tactile frame information on models with input features that span the pertinent physiological range. The finite element model has a dense surface mesh in order to provide detailed frames and minimize the problem of discrete jumps in the finite element information. The constitutive equations for the materials are fit to experimental data [7].

This work is supported by the US Army under grants DAMD17-01-10677 \& DAMD17-99-2-9001 

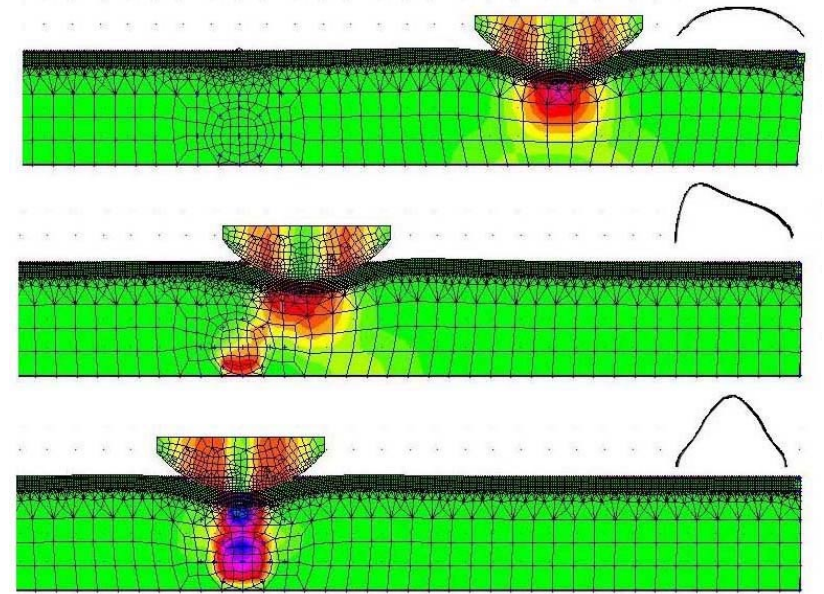

Figure 3. Finite element model used to obtain the pressure data. The model is symmetrical about the round lesion at the left of the image. The frames collected at each instance are shown on the right. They consist of the pressure information at the interface between the indentor and the tissue.

Previous work on tactile image inverting has resulted in algorithms for extracting the size and depth of a round inclusion but not the stiffness[7]. These approaches were limited by using a spatial average of the tactile frames. Our approach is to treat each frame as a discrete tactile image, and perform a least-squares fit to map a dense sampling of the frames to the four model features. In order to minimize noise in the data, we analyze each spatially unique frame by fitting a parabolic curve to the pressure data and extract four variables from each frame, namely peak pressure, edge pressure, width, and eccentricity. The latter is calculated as the asymmetry in the difference between each raw frame and a frame obtained far from the inclusion. Using this information we can invert the model more accurately than previous approaches.

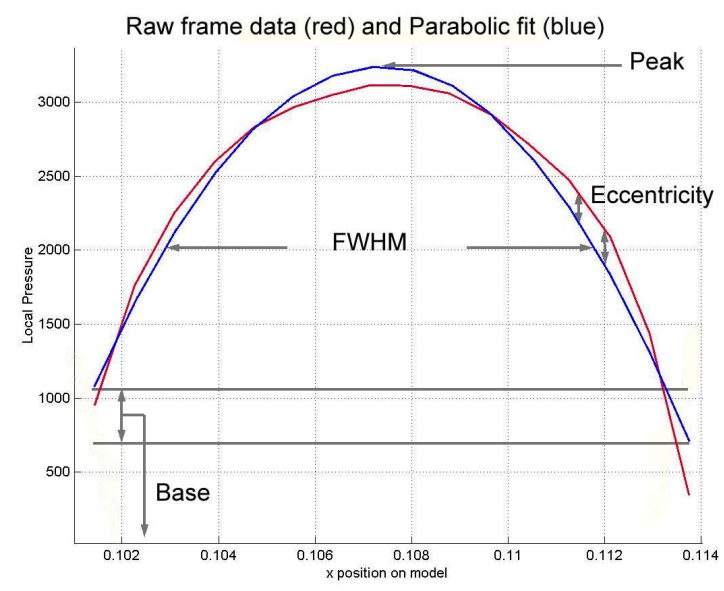

Figure 4. Sample frame and parabolic fit, showing extraction of the peak, base, FWHM, and eccentricity (calculated as the difference in symmetry between the current frame and a symmetrical frame obtained far from the inclusion).

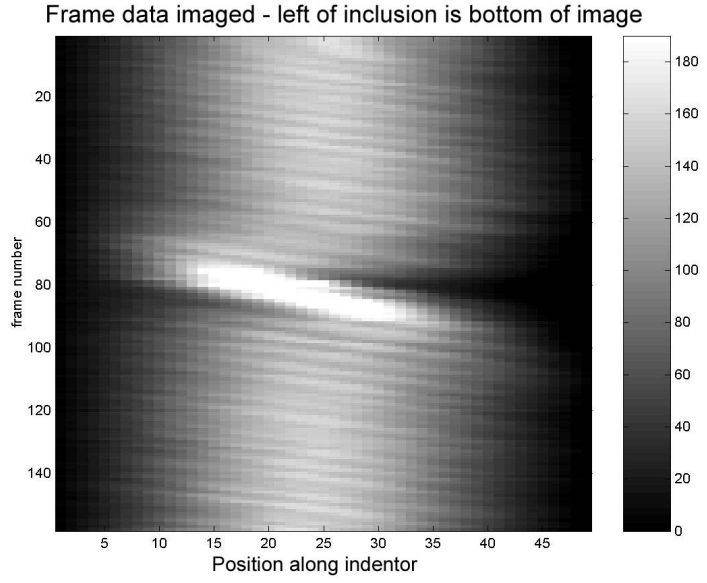

Figure 5. Frame data presented as a two-dimensional image. Each frame is shown horizontally, with the frames left of the inclusion at the bottom of the image. Note the eccentricity of the frames near the image, and their higher peak and lower base and FWHM values.

The stiffness information obtained using tactile imaging characterizes both healthy and diseased states [2,6]. In a clinical setting, tactile maps can be obtained on a patient in under a minute. The information our algorithm provides allows for quick assessment of patient tissue stiffness.

\section{REFERENCES:}

1. P. S. Wellman, R. D, Howe, N. Dewagan, M. A. Cundari, E. Dalton, K. A. Kern, "Tactile Imaging: A Method for Documenting Breast Masses," Proc. First Joint Biomed Eng Soc/IEEE Eng Med Bio Soc Conf, 1999:1131-2.

2. P.S. Wellman, E.P. Dalton, et al., "Tactile Imaging of Masses: First Clinical Report." Archives of Surgery, 136(2):204-08. Feb. 2001.

3. P.T. Lavin and G. Flowerdew. "Studies in variation associated with the measurement of solid tumors." Cancer. 1980; 46:1286-90.

4. Jatoi, Breast Cancer Screening. Medical Intelligence unit, Chapman \& Hall, New York: 1997

5. D.B. Kopans. "Clinical breast examination for detecting breast cancer." JAMA. 283(13):1688. 2000 Apr 5.

6. L. Ronnov-Jessen, O. Petersen, and M. Bissel. "Cellular changes involved in conversion of normal to malignant breast: importance of the stromal reaction." Physiological Reviews 76(1):69-125. 1996

7. P.S. Wellman, Tactile Imaging. Doctoral Thesis, Division of Engineering and Applied Sciences, Harvard University: May 1999 